\title{
Kirchhoff and Ohm in action: solving electric currents in continuous extended media
}

\author{
A E Dolinko ${ }^{1,2,3}$ (10) \\ ${ }^{1}$ Universidad de Buenos Aires, Facultad de Agronomía, Departamento de Ingeniería \\ Agrícola y Uso de la Tierra, Cátedra de Física, Buenos Aires, Argentina \\ ${ }^{2}$ Universidad de Buenos Aires, Facultad de Ciencias Exactas y Naturales, \\ Departamento de Biodiversidad y Biología Experimental, Buenos Aires, Argentina \\ ${ }^{3}$ CONICET-Universidad de Buenos Aires, Instituto de Micología y Botánica \\ (INMIBO), Buenos Aires, Argentina \\ E-mail: adolinko@df.uba.ar
}

Received 10 July 2017, revised 19 September 2017

Accepted for publication 5 October 2017

Published 9 January 2018

\begin{abstract}
In this paper we show a simple and versatile computational simulation method for determining electric currents and electric potential in 2D and 3D media with arbitrary distribution of resistivity. One of the highlights of the proposed method is that the simulation space containing the distribution of resistivity and the points of external applied voltage are introduced by means of digital images or bitmaps, which easily allows simulating any phenomena involving distributions of resistivity. The simulation is based on the Kirchhoff's laws of electric currents and it is solved by means of an iterative procedure. The method is also generalised to account for media with distributions of reactive impedance. At the end of this work, we show an example of application of the simulation, consisting in reproducing the response obtained with the geophysical method of electric resistivity tomography in presence of soil cracks. This paper is aimed at undergraduate or graduated students interested in computational physics and electricity and also researchers involved in the area of continuous electric media, which could find a simple and powerful tool for investigation.
\end{abstract}

Supplementary material for this article is available online

Keywords: electrical simulation, electrical resistivity distribution, photonics, electric resistivity tomography, soil cracks

(Some figures may appear in colour only in the online journal) 


\section{Introduction}

Several problems in physics involve the circulation of electric currents in complex or inhomogeneous extended media. For example, the circulation of electric currents in water bodies, the human body or in soils [1-3], or in a continuum bi-dimensional or three-dimensional space. It is also of interest to know the response of resistive or reactive complex electric circuits, as in the case of modelling of nanophotonics, photonic structures $[4,5]$ and metamaterials, by the application of a circuital model [6]. For that reason, it is useful to find a numerical simulation that easily allows evaluating the response of complex electric media.

In this work, we present a numerical simulation that allows us to reproduce the electric current density through a bi-dimensional space having an arbitrary resistive distribution. This paper could be relevant in the area of physics education since it shows the application of the basic laws of electric currents which are normally taught in courses of electricity, to scientific research to solve complex problems. The simulation model is based on a large rectangular grid of resistors. When the grid is very large, it can be considered as a continuum electric medium. The simulation is solved by an iterative algorithm that locally solves Kirchhoff's laws of electric currents combined with Ohm's law [7]. Then, we also show a generalisation of the method to three-dimensional spaces. A generalisation of the proposed simulation to describe the response of a continuous distribution of reactive impedance is also shown.

Finally, we show an example of application of the proposed method to study the response of the geophysical method of electric resistivity tomography (ERT) to soil cracks [8-10]. To do this, we reproduce data provided by the electrode configuration of a measurement performed with ERT for a synthetic soil profile (i.e. a bitmap) having cracks of known geometries. This method may be an alternative to classical approaches of forward modelling based on finite volume method as the one developed by Li and Oldenburg [11]. This is an important issue in soil physics and agricultural management since cracks play an important role in water evaporation and water availability in the soil. Because of that, it is interesting to investigate the ability of ERT to display the cracks in order to characterise them from a geometrical point of view.

\section{The physical model}

The electric simulation is based on a large rectangular circuit of $p \times q$ nodes contained in the $\mathbf{x}-\mathbf{y}$ plane separated each other by a distance $d_{n}$. Each node $n_{i j}$ for $i$ ranging from 1 to $p$ and $j$ ranging from 1 to $q$, is joined to their four nearest neighbours by four resistors $R_{i j}^{L}, R_{i j}^{R}, R_{i j}^{U}$ and $R_{i j}^{D}$, for the resistors located to the left, right, up and down of the node $n_{i j}$, respectively; as showed in figure 1.

If we consider the value of all the resistors in the network being the same, i.e. $R_{i j}=R$ $\forall i, j$; it can be quite easily demonstrated analytically that the equivalent resistance for a square section of the network (see figure 2 ) is $R_{\text {eq }}=R$. If we define the resistivity as

$$
\rho_{\text {sup }}=R_{\mathrm{eq}} d_{z} d_{n T} / d_{n}(\Omega \mathrm{m})
$$

where $R_{\text {eq }}$ is the equivalent resistance of the square section of the network considered, $d_{n T}$ is the transverse section, where we set $d_{z}=1$ (assuming translation symmetry in the $z$ direction perpendicular to the $\mathbf{x}-\mathbf{y}$ plane) and $d_{n}$ the length of this section, the resistivity of the network is $\rho_{\text {sup }}=R$. Therefore, in the limit $p, q \rightarrow \infty$, our large rectangular circuit can be considered as a continuous conducting medium with a resistivity $\rho_{\text {sup }}=R$. 


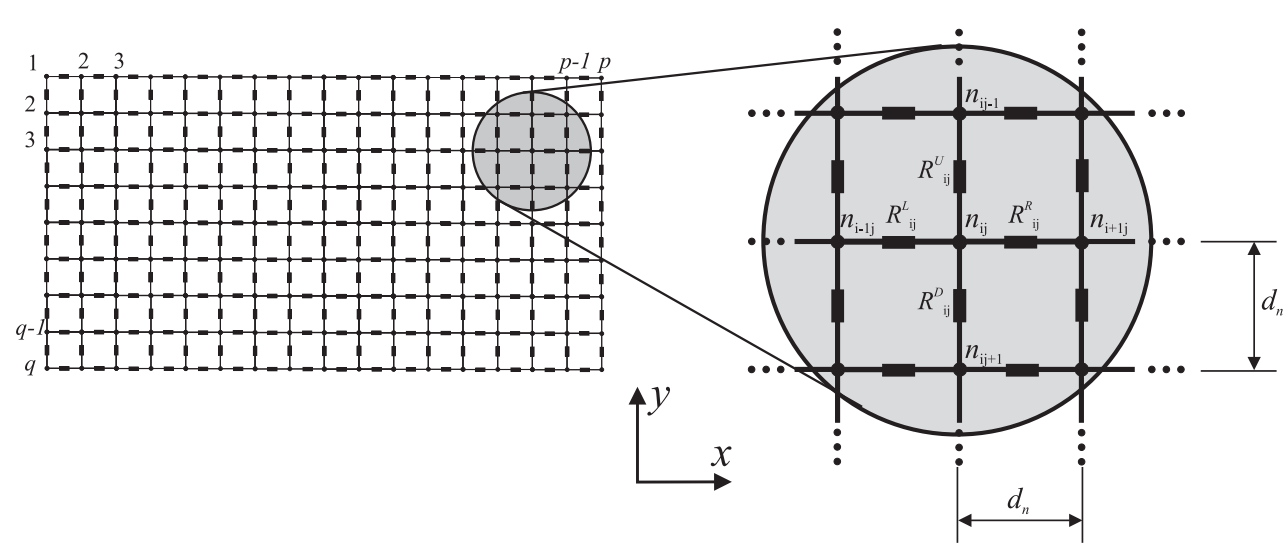

Figure 1. Electric circuit representing the physical system to be simulated.

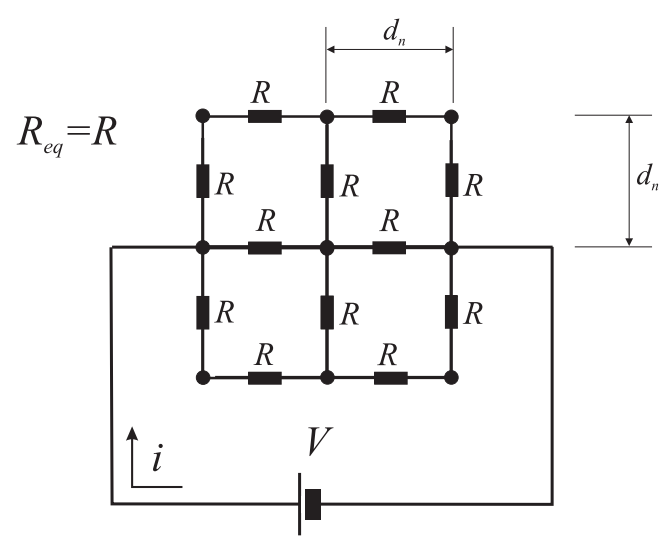

Figure 2. Schematic of the circuit used to find the equivalent resistance of a square section of the network (with $R$ being the resistors, $V$ the applied voltage and $i$ the total electric current).

\section{Description of the simulation}

\subsection{Defining the simulation space thorough digital images}

The simulation begins by defining a bi-dimensional space that is determined by a digital picture or bitmap $R_{\text {map }}$ with a size of $p \times q$ pixels. The bitmap defines a matrix with coordinates $(i, j)$ contained in the $\mathbf{x}-\mathbf{y}$ plane with $i=1,2, \ldots, p$ and $j=1,2, \ldots, q$. The elements $(i, j)$ of the matrix determine the position of nodes of the rectangular circuit, separated by a distance $d_{n}$ measured in metres, and joining 4 resistors, as displayed in figure 3 . So that each pixel $p_{i j}$ in the bitmap represents the position of a node $(i, j)$ and the grey level, ranging from 0 to 255 , codes the magnitude of the resistors. Similar methodology was implemented in heat and wave propagation computer simulations $[12,13]$. Since the pixels correspond to the position of the nodes and not to the resistors, the value of each resistor is taken as the average of the grey level of the pixels corresponding to the nodes it connects. That is as follows: $R_{i-1 j}=R_{c}\left(p_{i-1 j}+p_{i j}\right) / 2, R_{i+1 j}=R_{c}\left(p_{i j}+p_{i+1 j}\right) / 2, R_{i j-1}=R_{c}\left(p_{i j-1}+p_{i j}\right) / 2$ and 


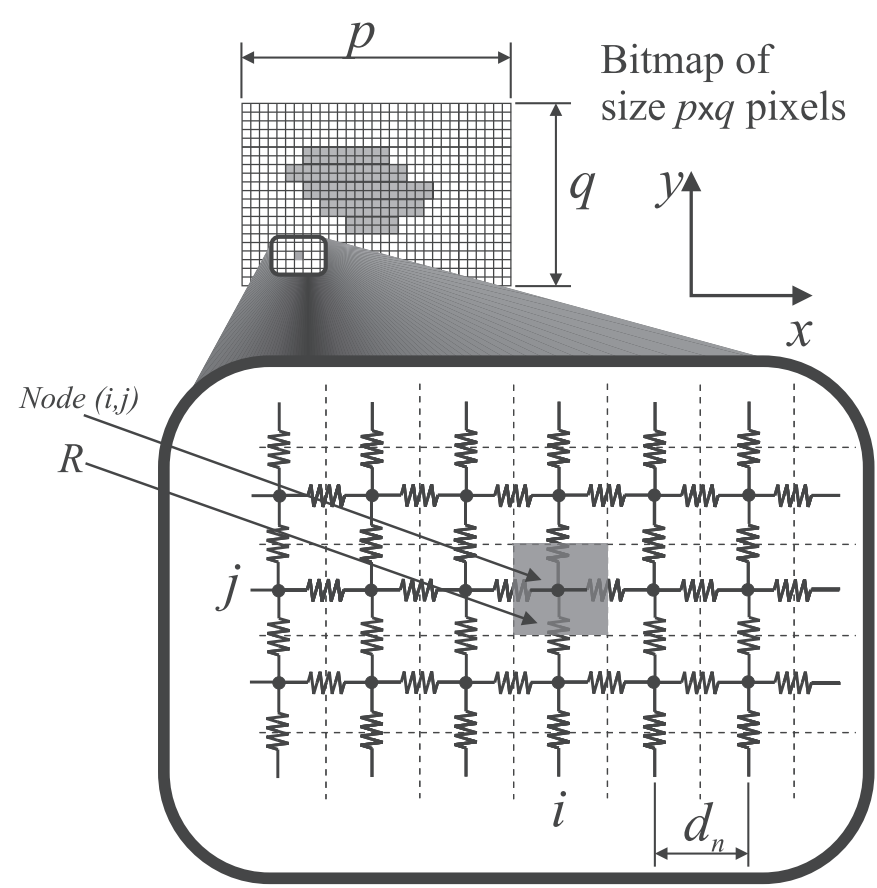

Figure 3. Interpretation of the bitmap $R_{\text {map. }}$

$R_{i j+1}=R_{c}\left(p_{i j}+p_{i j+1}\right) / 2$, where $R_{c}$ is a conversion factor linking the value of the grey level to adequate physical units in Ohms $(\Omega)$.

A second bitmap $V_{\text {map }}$ of the same size as $R_{\text {map }}$ codes in grey levels, the points of applied external voltage. This bitmap is polarised in the sense that a grey level of 127 corresponds to 0 volts, in such a way that a grey level over 127 (tending to white colour) corresponds to a positive applied voltage and a grey level under 127 (tending to black colour) corresponds to a negative applied voltage.

Therefore, two matrices $R_{\text {phys }}$ and $V_{\text {phys }}$ of size $p \times q$ containing the physical values of electric resistance and applied voltage are defined. These matrices are related to $R_{\text {map }}$ and $V_{\text {map }}$ as follows:

$$
\begin{aligned}
& R_{\text {phys }}=R_{c} R_{\text {map }}, \\
& V_{\text {phys }}=V_{c}\left(V_{\text {map }}-127\right),
\end{aligned}
$$

where $R_{\text {phys }}$ is the matrix determining the resistance in each point of the simulation space (in $\Omega), R_{\text {map }}$ is the bitmap containing the grey levels to be converted to resistance, $R_{c}$ is a proportionality constant in $\left(\Omega /\right.$ grey level), $V_{\text {phys }}$ is the matrix determining the applied voltage in each point of the simulation space (in Volts), $V_{\text {map }}$ is the bitmap containing the grey levels to be converted to applied voltage, and $V_{c}$ is a proportionality constant in (Volt/grey level).

\subsection{The algorithm of the simulation}

The simulation is based on a recursive scheme which obtains the resulting distribution of voltage similar to a relaxation method. Basically, the voltage at any node is calculated through a recursive equation from the voltage at the four neighbour nodes. A matrix of size $p \times q$ 


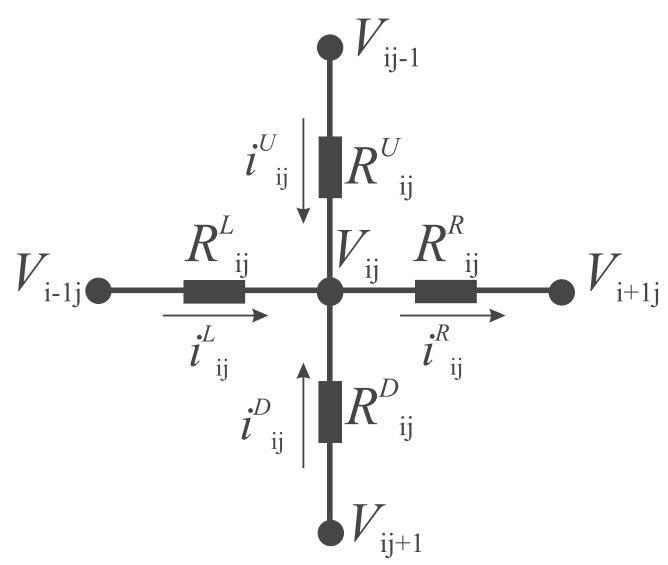

Figure 4. Kirchhoffs law for the electric current between the nodes $n_{i j}$ and $n_{i+1 j}$.

defining the voltage at each point (or pixel) of the simulation space ( $V_{i j}$ matrix) is initialised to zero, except those points corresponding to the external applied voltage, which are given by $V_{\text {phys }}$ matrix. This matrix is swept from left to right and from up to down to refresh the variables during an integer number of iterative loops $n$.

We adopt a convergence criterion to stop the iteration process. This criterion is based on evaluating the difference between the voltage mean values in the entire simulation space between two successive iteration steps, as follows:

$$
C_{v}=\operatorname{mean}\left(V_{n+1}\right)-\operatorname{mean}\left(V_{n}\right)
$$

where $C_{v}$ is the convergence value. When $C_{v}$ is lower than certain threshold value, the iteration is stopped. At this stage we assume that the final solution is obtained with a good approximation and reasonably low error.

We first find the recursive equation based in the Kirchhoff's law of electric currents [6] to be applied node by node of the circuit by sweeping all the elements of the matrix of size $p \times q$ from left to right and from up to down to update the variables during an integer number of loops $n$.

Setting the Kirchhoff's current law for node $n_{i j}$, we have (see figure 4):

$$
i_{i j}^{L}+i_{i j}^{U}+i_{i j}^{D}=i_{i j}^{R},
$$

where $i_{i j}^{L}, i_{i j}^{U}$ and $i_{i j}^{D}$ correspond to the electric current entering from the left, up and down of the node $n_{i j}$, respectively and $i_{i j}^{R}$ corresponds to the electric current coming out from the same node $n_{i j}$. The indices $(i, j)$ correspond to spatial dimensions $(x, y)$ respectively. By using Ohm's laws [7], (5) takes the following form:

$$
\begin{aligned}
& \left(V_{i j}-V_{i-1 j}\right) / R_{i j}^{L}+\left(V_{i j}-V_{i j-1}\right) / R_{i j}^{U}+\left(V_{i j}-V_{i j+1}\right) / R_{i j}^{D} \\
& \quad=\left(V_{i+1 j}-V_{i j}\right) / R_{i j}^{R} .
\end{aligned}
$$

By rearranging (6) and solving for $V_{i j}$, we obtain the recursive equation:

$$
\begin{aligned}
& V_{i j}=\left(V_{i+1 j} R_{i j}^{L} R_{i j}^{U} R_{i j}^{D}+V_{i-1 j} R_{i j}^{R} R_{i j}^{U} R_{i j}^{D}+V_{i j-1} R_{i j}^{L} R_{i j}^{R} R_{i j}^{D}+V_{i j+1} R_{i j}^{L} R_{i j}^{R} R_{i j}^{U}\right) \ldots \\
& \quad \ldots /\left(R_{i j}^{L} R_{i j}^{U} R_{i j}^{D}+R_{i j}^{R} R_{i j}^{U} R_{i j}^{D}+R_{i j}^{L} R_{i j}^{R} R_{i j}^{D}+R_{i j}^{L} R_{i j}^{R} R_{i j}^{U}\right) .
\end{aligned}
$$




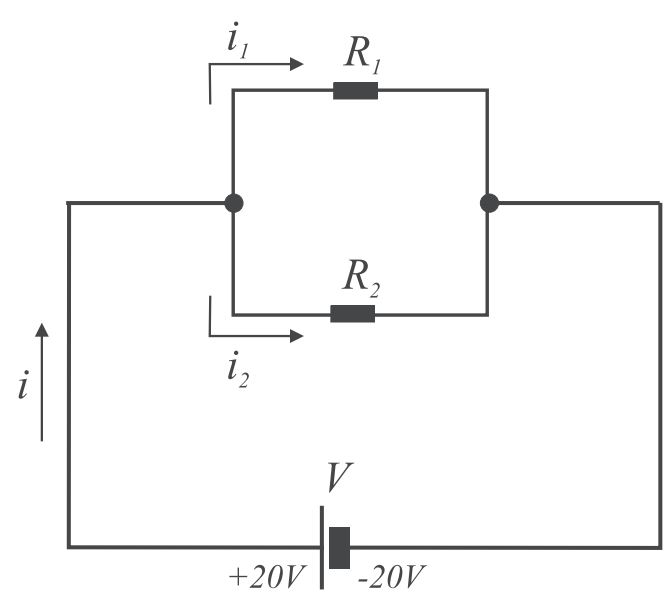

Figure 5. Circuit used to validate the simulation consisting in two resistors of the same value connected in parallel.

Equation (7) is used to sweep the matrix in order to find the final distribution of electric potential. After the ending of each iteration cycle, the values of applied external voltage over the resulting $V_{i j}$ matrix are forced in the locations determined by matrix $V_{\text {phys }}$.

Once the iterations have finished, we have the distribution of electric potential, the vector electric field is found as $E(x, y)=\nabla V(x, y)$, with in discrete form is expressed as

$$
E_{i j}=\nabla V_{i j}
$$

where $E_{i j}$ is a matrix having the discrete electric field and $\nabla^{n}$ is the discrete gradient. Then, using (8) and differential Ohm's law, we find the current density vector field as $J(x, y)=\sigma(x, y) E(x, y)$, where $\sigma(x, y)=1 / \rho(x, y)$, being the electric conductivity and $\rho(x, y)$ the resistivity map, in which the discrete form is

$$
J_{i j}=\sigma_{\mathrm{phys}} E_{i j} .
$$

Equation (9) is obtained by multiplication element by element of the involved matrices and the term $\sigma_{\text {phys }}=1 / R_{\text {phys }}$ is the matrix corresponding to the discrete form of the electrical conductivity $\sigma(x, y)$, where the matrix division is also element by element. Due to the finite size of the simulation space, boundary conditions are to be specified. The boundary conditions that better represent an infinite space are obtained by setting a very high value of resistivity in the borders of the map (tending to infinite), in relation to the average resistivity value of the rest of the map. In this manner, the leaking of electric current outside the simulation space is prevented.

\subsection{Numerical example and validation of the simulation}

In order to validate the simulation, we perform two tests. The first one evaluates the Kirchhoff laws over a canonical profile showing a simple circuit. Figure 5 shows the circuit used to validate the simulation consisting in two resistors of the same value (approximately $29 \Omega$ each) connected in parallel. The applied voltage is $40 \mathrm{~V}$. Figure 6(a) shows the bitmap representing the circuit depicted in figure 5 for the simulation, corresponding to a plate of 200 $\mathrm{mm} \times 120 \mathrm{~mm}$ and $1 \mathrm{~mm}$ of thickness. Figure $6(\mathrm{~b})$ shows the bitmap with the points of applied voltage according to the codification used in the simulation and explained in 
(a)

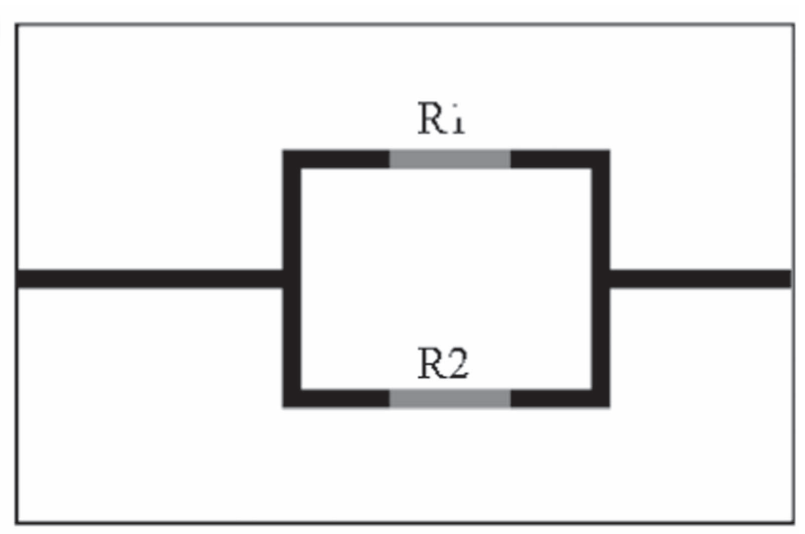

(b)

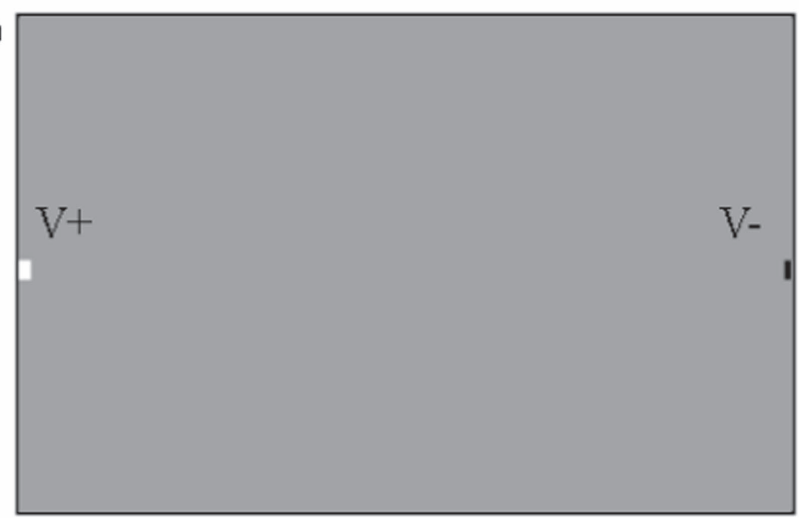

Figure 6. (a) The bitmap representing the circuit showed in figure 5 for the simulation, corresponding to a plate of $200 \mathrm{~mm} \times 120 \mathrm{~mm}$ and $1 \mathrm{~mm}$ of thickness; and (b) bitmap with points of applied voltage according to the codification used in the simulation.

section 3.1. The medium has a very high resistivity of $3 \times 10^{5} \Omega \mathrm{mm}$, so that it can be considered as an insulator around the conducting circuit. The conductors have a width of $5 \mathrm{~mm}$ and a resistivity of $1.18 \Omega \mathrm{mm}$. The resistors have the same width, a length of $31 \mathrm{~mm}$ and a resistivity of $4.72 \Omega \mathrm{mm}$, which by (1) lead to a total resistance of $29.26 \Omega$ for each resistor. The test consist in verifying the Kirchhoff law of conservation of currents, i.e. current in branch $1\left(i_{1}\right)$ plus current in branch $2\left(i_{2}\right)$ must be equal to the current going out the battery (i), which also must be equal to the current returning to the battery. It is worthwhile mentioning that the calculation of the electric current in the simulation is an indirect calculation obtained by integrating the electric current density obtained by means of (9) from the electric potential provided directly by the simulation.

Figure 7 shows the obtained map of electric current density for the resistivity maps describing the circuit and showed in figures 6(a) and (b). Figure 7 also shows the measurement points for the currents by integrating the current density along the width of the conductors, to measure the total intensity $(i)$ in point $\mathrm{A}$, current $i_{1}$ in point $\mathrm{B}$, current $i_{2}$ in point $\mathrm{C}$ and the current returning to the battery in point $\mathrm{D}$. Current in point $\mathrm{A}$ gives $0.8601 \mathrm{~A}$, in $\mathrm{B}$ and $\mathrm{C}$ gives $0.4306 \mathrm{~A}$ and $0.4318 \mathrm{~A}$, respectively. We have that $i_{1}+i_{2}$ gives $0.8624 \mathrm{~A}$, which corresponds very well with the total current $i$ in point A, verifying the Kirchoffs law 


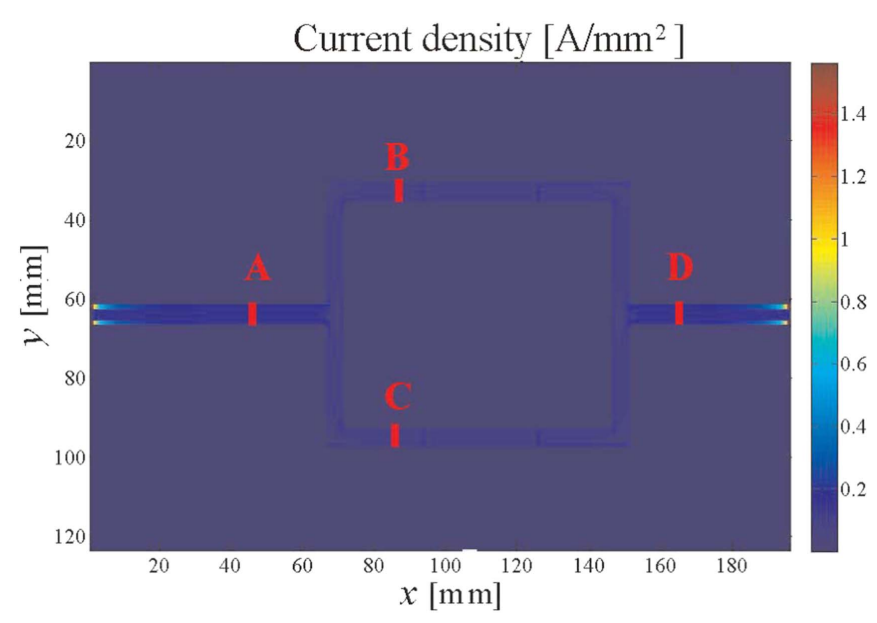

Figure 7. The obtained map of electric current density for the resistivity maps describing the circuit and points of current measurement (input current: A, branch 1: B, branch 2: C, output current: D).

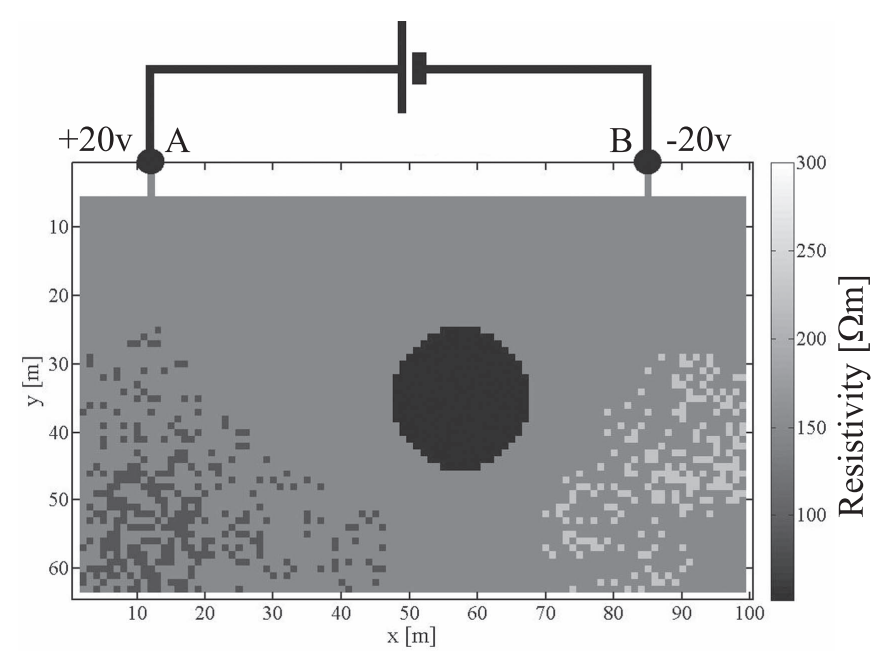

Figure 8. Resistivity map used to perform the second numerical validation of the simulation.

with a relative error of $0.23 \%$. Electric current in $\mathrm{D}$ is $0.8604 \mathrm{~A}$, which is very similar to the current in point $\mathrm{A}$, as one should expect.

The second test implies evaluating the electric potential on an arbitrary resistivity media due to the application of a difference of potential in two points. Then, we calculate the density of electric current and integrate it along the width of each electrode to obtain the magnitude of the electric current. By the Kirchhoff laws of currents, the electric current entering the media must be equal to the magnitude of the electric current that exit the media.

Figure 8 shows an arbitrary resistivity map used to perform the numerical validation of the simulation. It corresponds to a large bi-dimensional space of approximately $100 \mathrm{~m}$ long by $60 \mathrm{~m}$ of thickness (a bitmap size of $300 \times 190$ pixels, i.e. about 57000 nodes), with a 
(a)

Electric current lines and density $\left[\mathrm{A} / \mathrm{m}^{2}\right]$

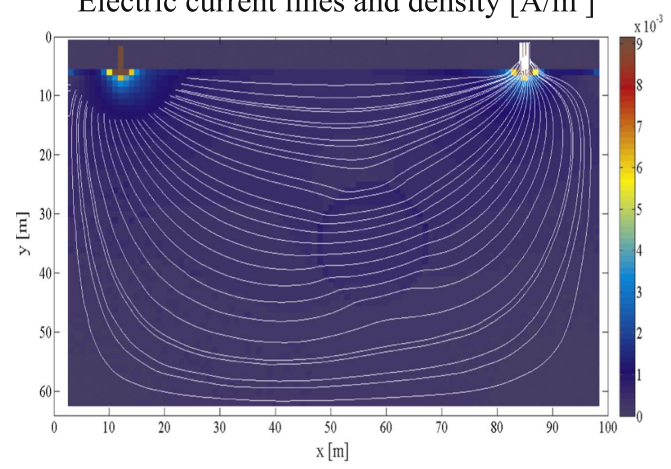

(b)

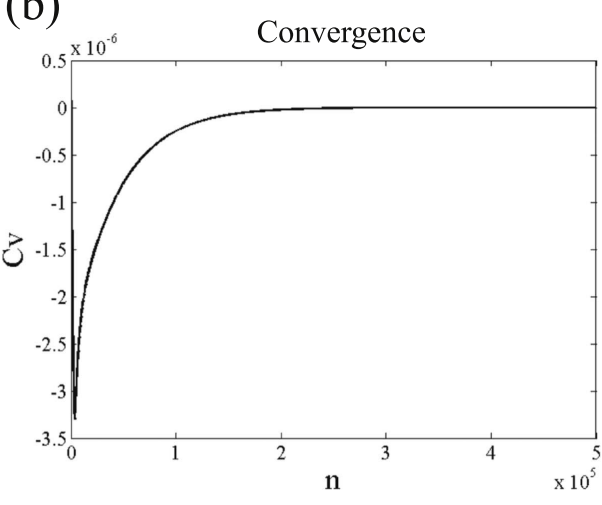

Figure 9. (a) Electric current flux lines and distribution of electric current density; and (b) the convergence parameter $C_{v}$.

resistivity ranging from 0 to $300 \Omega \mathrm{m}$, as showed by the grey colour scale. This region could represent a section of soil in depth. The regions of applied voltage are $+20 \mathrm{~V}$ in point $\mathrm{A}$ and $-20 \mathrm{~V}$ in point $\mathrm{B}$. The black circle represents a region of very low resistivity, which may represent a circular metal object beneath the surface in the resistive background, having a resistivity value of $1885 \Omega \mathrm{m}$. A region of conductive noise is also added on the left side of bitmap (sparse black pixels) and a region of non-conductive noise on the right side (sparse white pixels).

Figure 9(a) show the results obtained with the simulation for the bitmaps showed in figure 8. It can be noticed that the electric current flux lines tend to be concentrated over the conductor circle depicted in the resistivity bitmap, as expected. It can also be noticed that the lines tends to cover the conductive region (sparse black pixels in figure 8 on the left side of the simulation space) and avoid the right side, having the sparse pixels with higher resistivity. The input current in point $\mathrm{A}$ is $11.5 \mathrm{~mA}$, while the output current is $11.4 \mathrm{~mA}$, corresponding to a relative error of $0.86 \%$ for the second test. Figure 9 (b) shows the evolution of the convergence parameter $C_{v}$ (see (4)) for the test showed in figure 9(a). The simulation was run for $n=5 \times 10^{5}$ iterations, and according to the convergence parameter and from figure 9(b), it can be verified that in this case, the simulation reaches stability after $n=2 \times 10^{4}$ iterations approximately. The number of iterations to reach convergence depends on the size in pixels of the bitmap defining the simulation space. Smaller number of iterations to convergence can be obtained by reducing the resolution of the simulation space (i.e. by reducing the size in pixels of the bitmap).

\subsection{Generalisation to $3 D$ configuration}

Similarly to section 3.2, we set the Kirchhoff current law for for node $n_{i j k}$, were now indexes $(i, j, k)$ correspond to spatial dimension $(x, y, z)$ respectively, we have (see figure 10):

$$
i_{i j}^{L}+i_{i j}^{U}+i_{i j}^{D}+i_{i j}^{F}+i_{i j}^{B}=i_{i j}^{R},
$$

where here $i_{i j k}^{L}, i_{i j k}^{U}, i_{i j k}^{D}, i_{i j k}^{B}$ and $i_{i j k}^{F}$ correspond to the electric current entering the node $n_{i j k}$ from the left, up, down, behind and front, respectively, and $i_{i j k}^{R}$ corresponds to the electric current coming out from the same node $n_{i j k}$. After some mathematical manipulation similar to the procedure described in section 3.2 , we obtain de recursive equation in $3 \mathrm{D}$ : 


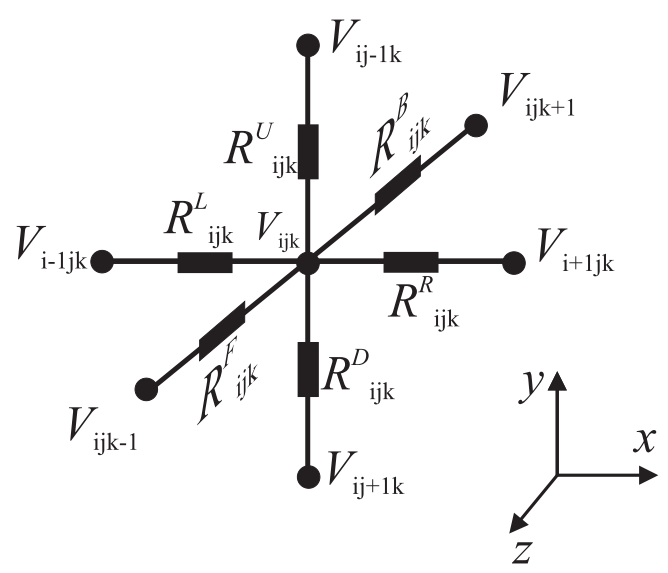

Figure 10. Kirchhoff law for the electric current between the nodes $n_{i j k}$ and $n_{i+1 j k}$ for the $3 \mathrm{D}$ configuration.

$$
\begin{aligned}
V_{i j k}= & \left(V_{i+1} R^{L} R^{B F} R^{U D}+V_{i-1} R^{R} R^{B F} R^{U D}+\ldots\right. \\
& V_{j+1} R^{U} R^{L R} R^{B F}+V_{j-1} R^{D} R^{L R} R^{B F}+\ldots \\
& \left.V_{k+1} R^{F} R^{L R} R^{U D}+V_{k-1} R^{B} R^{L R} R^{U D}\right) / \ldots \\
& \left(R^{L} R^{B F} R^{U D}+R^{R} R^{B F} R^{U P}+R^{U} R^{L R} R^{B F}+\ldots\right. \\
& \left.R^{D} R^{L R} R^{B F}+R^{F} R^{L R} R^{U D}+R^{B} R^{L R} R^{U D}\right),
\end{aligned}
$$

where we adopted the following compact notation consisting in writing only incremented or decremented indices for voltages:

$$
V_{i+1}=V_{i+1 j k},
$$

taking out the indexes for resistors as follows

$$
R^{L}=R_{i j k}^{L},
$$

and grouping the $R$ 's appearing in each term at both sides for the same dimension as follow:

$$
R^{L R}=R_{i j k}^{L} R_{i j k}^{R},
$$

and the same for resistors along the $\mathbf{y}$ and $\mathbf{z}$ dimensions.

The $3 \mathrm{D}$ resistivity distribution is generated by means of slices of $2 \mathrm{D}$ resistivity maps obtained from images, as described in section 3.1, or by mean of inequalities in case of ellipsoids or spheres, depending of the symmetry of the 3D resistivity distribution to be generated. Figure 11 shows a numerical example for a 3D configuration of resistivity of a medium with a resistivity of $300 \mathrm{~m} \Omega$, with two parallel conducting plates $10 \Omega \mathrm{m}$, having different longitudes.

\subsection{A generalisation to reactive configuration}

The reactive configuration is obtained by adding to each resistor $R_{i j}^{L}$ (measured in Ohms $(\Omega)$ ), a capacitor $C_{i j}^{L}$ (measured in in Farads $\left.(\mathrm{F})\right)$ and an inductance $L_{i j}^{L}($ measured in Henries $(\mathrm{H}))$ in parallel connection, as showed in figure 12 . The equivalent inductive resistivity $Z_{i j}^{L}$ (i.e. we define the inductive resistivity as the intensive magnitude associated to the inductance) as a function of the angular frequency $\omega$ (in $\left(\operatorname{radian~} \mathrm{s}^{-1}\right)$ ) is calculated as the parallel among $R_{i j}^{L}$, the reactive capacitance $X_{C i j}^{L}=-\mathrm{i} \omega C_{i j}^{L}$, and the reactive inductance $X_{L i j}^{L}=\mathrm{i} \omega L_{i j}^{L}$, where $\mathrm{i}$ is 

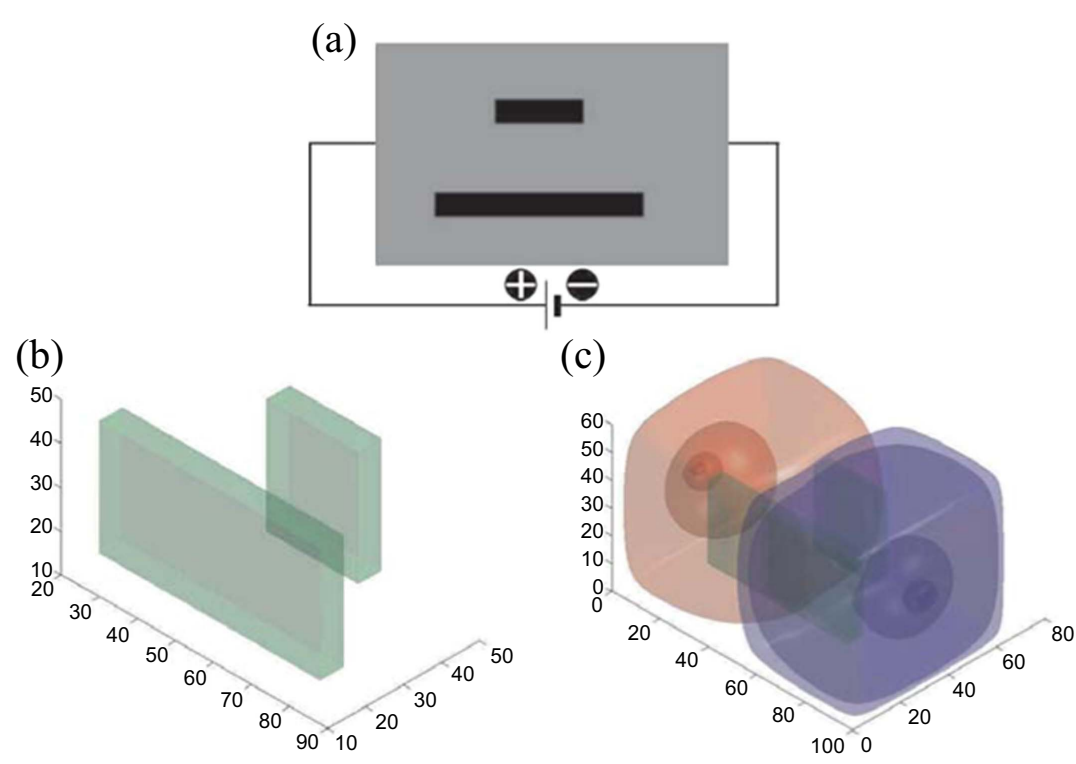

Figure 11. Numerical example of the 3D version of the simulation: (a) bitmap used to generate two parallel conducting plates in the medium and the position of the applied voltage corresponding to the mid height of the simulation space; (b) 3D representation of the conducting plates and (c) 3D equipotentials obtained with the simulation (red for $V \geqslant 0$, blue for $V<0$ and the plates in green).
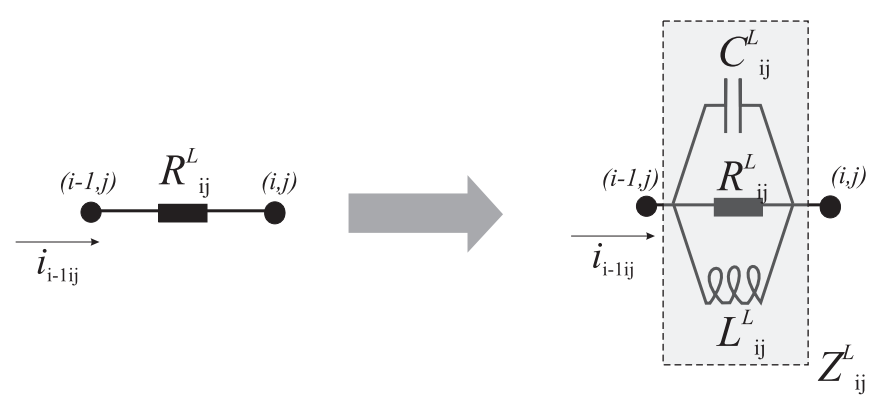

Figure 12. Schematic representation of the modification of the circuit from purely resistive to reactive.

the imaginary unit. Therefore, the equivalent inductive resistivity $Z_{i j}^{L}$ is expressed as

$$
Z_{i j}^{L}(\omega)=1 /\left(1 / R_{i j}^{L}+1 / X_{C i j}^{L}+1 / X_{L i j}^{L}\right) .
$$

So that, for the reactive configuration, each resistor $R_{i j}^{L}$ in (7) is replaced by the parallel equivalent inductance $Z_{i j}^{L}$, being expressed as

$$
\begin{aligned}
& V_{i j}(\omega)=\left(V_{i+1 j} Z_{i j}^{L} Z_{i j}^{U} Z_{i j}^{D}+V_{i-1 j} Z_{i j}^{R} Z_{i j}^{U} Z_{i j}^{D}+V_{i j-1} Z_{i j}^{L} Z_{i j}^{R} Z_{i j}^{D}+V_{i j+1} Z_{i j}^{L} Z_{i j}^{R} Z_{i j}^{U}\right) \ldots \\
& \quad \ldots /\left(Z_{i j}^{L} Z_{i j}^{U} Z_{i j}^{D}+Z_{i j}^{R} Z_{i j}^{U} Z_{i j}^{D}+Z_{i j}^{L} Z_{i j}^{R} Z_{i j}^{D}+Z_{i j}^{L} Z_{i j}^{R} Z_{i j}^{U}\right) .
\end{aligned}
$$

In this case, the data is loaded by means of three bitmaps, giving the resistivity map, the capacity map and inductance map. It is worthwhile to mention that this is a simple 


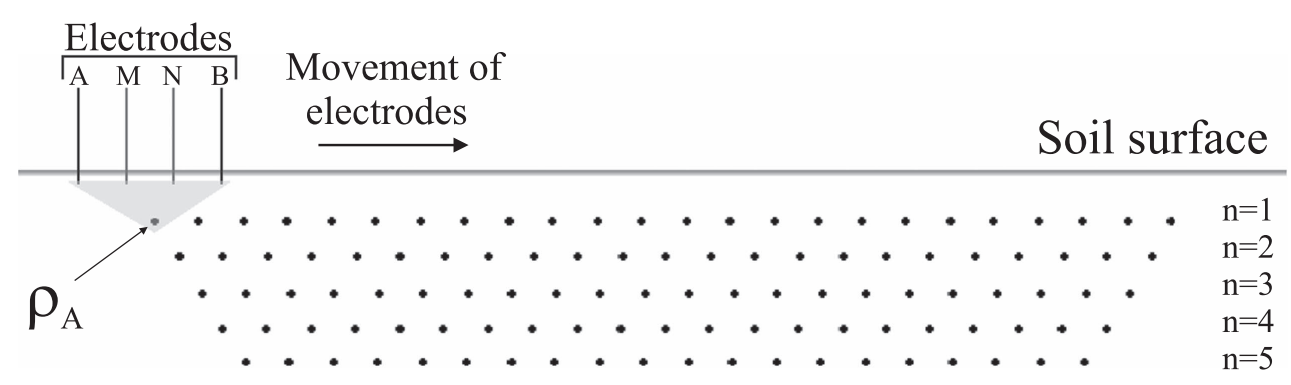

Figure 13. Measurement of apparent resistivity along the transect and in depth.

formulation that does not take into account the mutual inductance among the solenoids of the circuit, so that, effects as the Eddy currents will not be reproduced by this simulation in the present form. Future work will include this feature.

\section{Example of application to electrical resistivity tomography (ERT)}

In order to show the application of the proposed method in scientific research where complex conducting media are involved, we present an example of the application of the electric simulation to investigate the response of a geophysical method called ERT to soil cracks.

The ERT is a noninvasive technique that displays an estimate of the distribution of resistivity in the earth beneath the surface. Bi-dimensional models of resistivity are obtained laterally and through depth and in the case of near surface studies, for example the first metres of depth, they are related with water content, clay content and salinity of solution inside the pores of the soil. Among other things, this methodology allows to identify the presence of cracks in the soil, due to the resistivity contrast they show in relation to the background $[10,11]$. The ERT method generally consists in the use four electrode probes. Two electrode probes are used to inject a known current in the soil and the other two electrode probes are used to measure the resulting difference of electric potential. Assuming a homogeneous resistivity distribution of the soil, the apparent resistivity is estimated along a lateral profile and at a depth that depends on the separation of the probes [8]. By repeating the procedure along a line over the surface of the soil and for different probe separations, it is obtained a pseudo-section of apparent resistivity. There exists several configurations for the current and voltage measurement of the electrode probes. In this case, we used the Wenner configuration, consisting in injecting current in the outward electrodes (generally denoted as electrodes A and $\mathrm{B}$ ) and measuring the resulting voltage in the inward electrodes (denoted as $\mathrm{M}$ and $\mathrm{N}$ ). The distance between electrodes denotes the position of the apparent resistivity and it is calculated as [8]:

$$
\rho_{A}=k_{G} V / i
$$

where $k_{G}$ is a geometric factor which depends on the distance between electrodes and the type of electrode configuration. For each lateral measurement, the four electrodes A, B, M, N, are shifted along the transect, which give a value of apparent resistivity $\rho_{A}$ for each position. Depth scanning ( $n=1, n=2$, ) is carried out by increasing the distance between electrodes [8] (see figure 13). Then, experimental data may be processed by software of inversion such as DCIP2D code (from UBCGIF) [14], which provides a cross-section or 'pseudo'-section of the resistivity through the ground. The code follows the approach from Oldenburg and Li [7] 


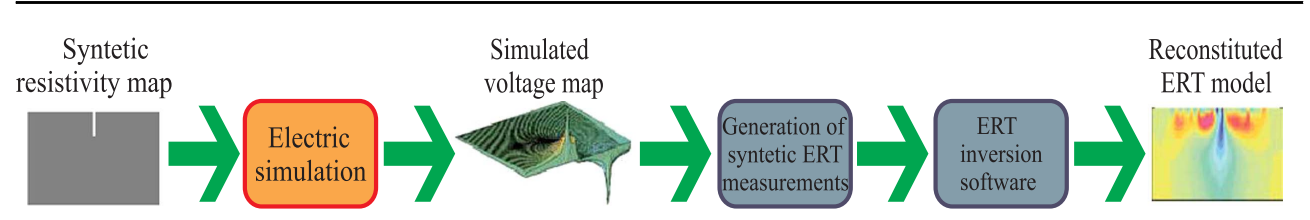

Figure 14. Process to obtain a simulated ERT 'pseudo'-section from a synthetic resistivity profile of soil.

(a)
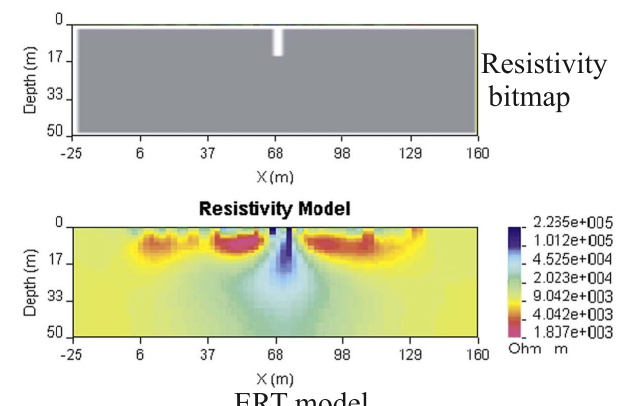

(b)
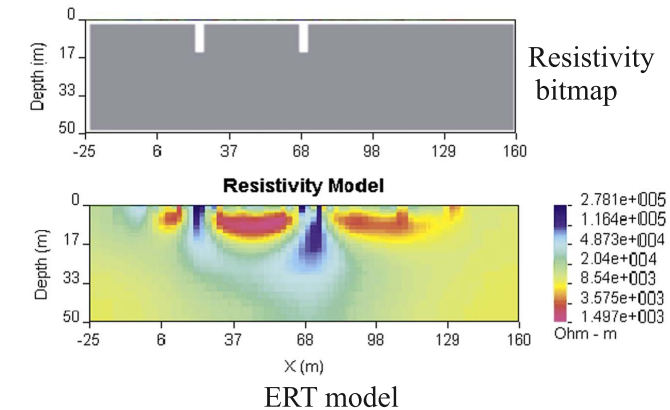

Figure 15. Bitmaps representing soil cracks and the corresponding ERT model obtained by means of inversion with DCIP2D of the synthetic data obtained with the electrical simulation for a soil profile having (a) a single crack; and (b) two cracks.

which finds a model minimising an objective function subject to fitting the data to a specified degree $[15,16]$.

The ERT method is widely used to explore groundwater resources and studies of soil contamination $[17,18]$ but it is not proved to reveal the exact morphology of cracks in the soil with a size of the order of metres or centimeters, which is very important in agronomy to determine the availability of water and water evaporation in the soil, for example. Therefore, it is interesting to investigate the ability of ERT to display the cracks in order to characterise them. The aim is to define certain indexes indicating parameters of interest, such as depth, thickness and air volume of detected cracks. However, it becomes experimentally difficult to produce cracks of known profiles to analyse the response of ERT.

For this reason, the proposed computer simulation method was used to analyse the ERT method by introducing cracks of known profiles with specific length and thickness parameters [19]. The simulation is used to synthetically reproduce the apparent resistivity data (forward problem) that would be experimentally measured by an ERT survey on the soil surface, with configuration dipole-dipole or Wenner, for bitmaps representing soil profiles having canonical crack geometries. These results are then inverted with the programme DCIP2D to obtain the model of the cracks. Figure 14 shows schematically the process for obtaining a simulated ERT 'pseudo'-section from a synthetic resistivity profile of soil. It is worthwhile to mention that the process for an experimental ERT measurement, begins with the feeding of the ERT inversion software with experimental ERT measurements, in place of synthetic ERT measurements, generated in this case with the proposed simulation.

Figure 15 shows the results of ERT output obtained from the inversion of synthetic ERT data with Wenner configuration obtained with the simulation for two bitmaps representing a soil section having $160 \mathrm{~m}$ length and $50 \mathrm{~m}$ depth having one (a) and two (b) superficial cracks 
of around $8 \mathrm{~m}$ depth. The bitmaps used for the simulation for cases (a) and (b) are shown in the upper part of the figure. As explained above, the bitmap codification is such that white pixels are those with higher resistivity values and dark pixels correspond to lower values of resistivity. The value of simulated background resistivity is $1885 \Omega \mathrm{m}$ (grey colour in the bitmaps). It is clear that the ERT model obtained with DCIP2D from the results obtained with the simulation represent very well the position of the cracks (in blue colour).

\section{Conclusion}

This paper presents an electrical simulation based on a large arrangement of resistors. Due to the size of the electric network of resistors, it can be considered as a continuous bi-dimensional electric medium. The simulation allows reproducing the electric potential and distribution of current density for an arbitrary distribution of resistivity. One of the highlights of the proposed simulation is that the map of resistivity profile to be simulated can be easily fed to the simulation by means of digital images or bitmap. A validation of the simulation was presented. Its generalisation to three-dimensional spaces and reactive media (i.e. having capacitive and inductive materials) was also carried out. For the last case, a future version of the simulation will take into account the mutual inductance among the solenoids in order to enlarge the phenomena that will be able to be simulated and be able to simulate effects such as eddy currents. Finally, an example of the application of the proposed simulation for the study of the response of the ERT method to characterise soil cracks was carried out. The simulation of the response of cracks of vertical geometries proved to be a good approximation for characterising the shape and dimension of the soil cracks having a few metres in depth. Future work will aim to study the response of the ERT method for the detection and characterisation of different geometries of soil cracks. The 3D version presented in this work will also allow extending this study to 3D geometries, while the inductive version will be studied to investigate the development of new kinds of ERT methods accounting for inductive phenomena in the soil.

\section{Acknowledgments}

The author would like to thank the Consejo Nacional de Investigaciones Científicas y Técnicas of Argentina (CONICET) and University of Buenos Aires for providing the financial support. The author also wishes to thank Professor Dr Claudia Sainato and Professor Mg Beatriz Losinno for their valuable comments and critical reading of the manuscript.

\section{ORCID iDs}

A E Dolinko (10 https://orcid.org/0000-0002-5111-3924

\section{References}

[1] Dam J V and Meulenkamp J J 1967 Geophys. Prospect. 15 92-115

[2] Deno D W 1977 IEEE Trans. Power Appar. Syst. 961517

[3] Corwin D L and Lesch S M 2005 Comput. Electron. Agric. 4611

[4] Dolinko A E and Skigin D C 2013 J. Opt. Soc. Am. A 301746

[5] Inchaussandague M E, Skigin D C and Dolinko A E 2017 Appl. Opt. 565112 
[6] Marques R, Martin F and Sorolla M 2008 Metamaterial with Negative Parameters (Hoboken: Wiley)

[7] Alexander C and Sadiku M 1999 Fundamentals of Electric Circuits 6th edn (New York: McGraw-Hill)

[8] Telford W M, Geldart L P and Sheriff R E 1990 Applied Geophysics (Cambridge: Cambridge University Press)

[9] Abbas I M 2015 Int. J. Eng. Tech. Res. 3256

[10] Tabbagh J, Samouëlian A, Tabbaghand A and Cousin I 2007 J. Appl. Geophys. 62313

[11] Li Y and Oldenburg D W 2000 Geophysics 651931

[12] Dolinko A E 2008 J. Phys. D: Appl. Phys. 41205503

[13] Dolinko A E 2009 Eur. J. Phys. 301217

[14] Li Y and Oldenburg D W 2001 Inversion for Applied Geophysics: a tutorial, UBC-Geophysical Inversion Facility, Department of Earth and Ocean Sciences, University of British Columbia, Vancouver, Canada http://eos.ubc.ca/research/ubcgif/iag/tutorials/tutorial-v9.pdf

[15] Oldenburg D W, McGillivray P R and Ellis R G 1993 Geophys. J. Int. 114 12-20

[16] Oldenburg D W and Li Y 1994 Geophysics 59 1327-41

[17] Sainato C M, Losinno B N and Malleville H J 2010 Near Surf. Geophys. 8163

[18] Sainato C M and Losinno B N 2012 Real time electrical monitoring of the soil infiltration $A G U$ Fall-Meeting (San Francisco, USA)

[19] Dolinko A E, Losinno B N, Zunni N and Lemeillet F 2016 Analysis of the response of the electrical resistivity tomography method to soil cracks 22nd European Meeting of Enviromental and Engineering Geophysics (Barcelona, Spain) 